\title{
Is host lipidation of pathogen effector proteins a general virulence mechanism?
}

\author{
Stéphane Méresse ${ }^{1,2,3 *}$ \\ Centre d'Immunologie de Marseille-Luminy, Université de la Méditerranée, Marseille, France \\ 2 INSERM, U631, Marseille, France \\ ${ }^{3}$ CNRS, UMR6102, Marseille, France \\ *Correspondence: meresse@ciml.univ-mrs.fr
}

Many bacterial pathogens exploit secretion systems to convey virulence proteins, called effectors, into eukaryotic host cells. Often, these effector proteins are collectively necessary for the progression of infection. The molecular mechanisms by which these effectors support virulence have been the subject of much research. Recent papers analyzing the intracellular fate of Legionella pneumophila effectors have underlined the importance of their subcellular localization in challenging host cellular processes and underscored the exploitation of the eukaryotic lipidation machinery by bacterial pathogens.

The proper localization of proteins to specific subcellular compartments is crucial in the functioning of eukaryotic cells. This is also true and even more critical for prokaryotic proteins given that bacterial effectors are translocated into eukaryotic cells in small quantities. Thus, they need to be directed to their downstream target in order to be able to accomplish their functions.

Similar to eukaryotic proteins synthesized by free ribosomes, translocated effector proteins need to be transported to their intended host compartments and membranes. These include the nucleus (Zurawski et al., 2006), endoplasmic reticulum (Campodonico et al., 2005), plasma membrane (Schmid et al., 2006), or Golgi (Geddes et al., 2005) even though effectors are frequently found in close proximity to their translocation site (Kenny et al., 1997). Targeting of effectors to the cytoplasmic face of endo-membranes is possible owing the presence of hydrophobic domains (Salcedo and Holden, 2003) or the posttranslocation modifications by covalent attachment of lipid groups. These modifications facilitate membrane attachment of effectors, their subcellular targeting, and very likely their partitioning into specific membrane domains.

\section{PROTEIN LIPIDATION IN EUKARYOTIC CELLS}

The involvement of lipidation in some severe human diseases (cancer, genetic blindness, premature aging, or osteopetrosis; Perez-Sala, 2007) underlies the functional significance of these posttranslational modifications. Lipidation of proteins include modifications such as acylation (also called palmitoylation), myristoylation, and prenylation.

Prenylation of eukaryotic proteins consists of the covalent incorporation of an isoprenoid lipid to cysteine residues located at the carboxy terminus. Eukaryotic cells encode many proteins bearing cysteine as the fourth to last residue (Clarke et al., 1988). Indeed, a consensus sequence designated CAAX (C for cysteine, A for an aliphatic amino acid, and X for any amino acid) that ends the primary translation product has been shown to direct a series of posttranslational modifications that start by a prenylation. The lipid substrates, which are 15-carbon farnesyl or 20-carbon geranylgeranyl isoprenoids, are linked to the cysteine of the CAAX motif by thioether linkages. These prenylations are catalyzed by a farnesyltransferase (FTase) or a geranylgeranyltransferase (GGTase; Zhang and Casey, 1996). Prenylated proteins are further processed. The Ras-converting enzyme-1 (RCE-1) cleaves the last three amino acids (AAX) while the C-terminal isoprenyl cysteine is methylated by the isoprenyl cysteine carboxyl methyltransferase (ICMT; Michaelson et al., 2005).

\section{BACTERIAL EFFECTORS ARE LIPIDATED BY HOST ENZYMES}

The lipidation of bacterial effector proteins by the eukaryotic enzymatic machinery was first described for the phytopathogen Pseudomonas syringae
(Nimchuk et al., 2000). Upon translocation into plant cells, Avr effectors are cleaved and modified by $\mathrm{N}$-myristoylation and S-palmitoylation (Dowen et al., 2009). In 2003, we showed that the Salmonella effector protein SifA has a C-terminal CAAX motif, which is necessary and sufficient to target SifA or the green fluorescent protein to membranes (Boucrot et al., 2003). SifA is isoprenylated through the action of the geranylgeranyl transferase I (Reinicke et al., 2005).

In 2010, two independent groups published studies putting forward the idea that subversion of host lipidation by bacterial pathogens could represent a conserved virulence strategy. The Dot/Icm type IV secretion of L. pneumophila translocates AnkB. The group of Abu Kwaik established that host cell farnesylation anchors AnkB to the membrane of the Legionella vacuole, both in human macrophages and Dictyostelium discoideum (Price et al., 2010a; Al-Quadan and Abu Kwaik, 2011). Puzzlingly, host enzymes involved in prenylation (FTase) and subsequent processing (RCE-1 and ICMT) are recruited to the Legionella vacuole in a Dot/Icm-dependent manner. Whether farnesylated effectors are processed is not known but this is suggested by the presence of the necessary enzymes on the vacuole and the fact RNAi knockdown of these enzymes blocks targeting of AnkB to membranes.

Specific effector proteins could mediate the vacuolar recruitment of the lipidation enzymes. However, the slight but significant reduction in vacuolar localization of the FTase in absence of AnkB indicates a substrate-dependent recruitment (Price et al., 2010a,b). This hypothesis is sustained by the finding that $L$. pneumophila encodes $>10 \mathrm{Dot} / \mathrm{Icm}$ effector proteins bearing a functional C-terminal CAAX motif (Ivanov et al., 2010; Price et al., 2010b). Interestingly the homology between these proteins is 
limited to the CAAX motif, suggesting that proteins with different functions are targeted to membrane through prenylation.

\section{WHERE DOES PRENYLATION OCCUR?}

While farnesylation of eukaryotic proteins occurs in the cytosol (Wright and Philips, 2006), the FTase is recruited to the Legionella vacuole. Thus, it was proposed that farnesylation of effectors occurs at the bacterial vacuolar membrane (Price et al., 2010a). However AnkB might also be modified in the cytosol before being anchored to the vacuolar membrane as a substrate-enzyme complex. Both RCE-1 and ICMT are ER-resident proteins and the post-farnesylation processing of eukaryotic proteins occurs on the cytosolic surface of the ER membrane. As Legionella resides in a compartment derived from the ER, the vacuole may conceivably be a default compartment for the localization of RCE-1 and ICMT. Still, these enzymes are also recruited to the bacterial vacuole in a Dot/Icmdependent manner. It is not known whether the GGTase is recruited to the Salmonella vacuole to modify SifA.

\section{ROLE OF PRENYLATION IN EFFECTORS FUNCTIONS}

Using a point mutant form of AnkB, Price et al. (2010a) showed that farnesylation of AnkB is necessary for its anchoring to the Legionella vacuole and for its biological function, which has been described as the vacuolar docking of polyubiquitinated proteins. In the mouse model, this modification is also necessary for intrapulmonary proliferation. Not surprisingly inhibition of the aforementioned enzymes results in loss of biological function of AnkB. Results are more contrasted for the Salmonella effector SifA. As far as the replication in macrophages and virulence in mice are concerned, we established that the C-terminal hexapeptide of SifA, which includes the CAAX motif, is required (Boucrot et al., 2003), while Reinicke et al. (2005) found that strains expressing point mutant forms of SifA deficient in S-acylation or prenylation were comparable to the wild-type strain. A differential vacuolar recruitment of these mutant forms of SifA could explain these discrepancies. Though a general rule cannot be established, it is likely that lipidation promotes activity of bacterial effectors by facilitating the interaction with host targets or other effectors.

\section{MANY PATHOGENS ENCODE C-TERMINAL CAAX MOTIF-CONTAINING PROTEINS}

As demonstrated by the groups of Roy (Ivanov et al., 2010) and Abu Kwaik (Price et al., 2010b) several Legionella strains encode a number of CAAX motifcontaining proteins and most of them are effectors. Thus, an important question raised is whether the exploitation of host lipidation machinery is a widely used virulence strategy. Price et al. (2010a) searched for CAAX motif-containing proteins by in silico analyses of bacterial pathogens capable of injecting effector proteins into eukaryotic cells. Proteins harboring a C-terminal prenylation motif were found in about 20 bacterial pathogens but not in non-pathogenic bacteria. Remarkably this motif was rarely found in products of housekeeping genes. This suggests that the presence of a functional CAAX on virulence proteins results from the adaptation of the pathogen to the host, and indirectly that a CAAX motif is a hallmark of effector proteins.

Collectively, these studies illustrate how bacterial pathogens exploit a host-targeting signal to localize translocated virulence factors to specific eukaryotic membranes. Considering the great number bacterial pathogens encoding CAAX motif proteins, the functional significance of this posttranslocation modification will certainly be an area of active investigation. Most interestingly, by limiting the function of these proteins, lipidations enzymes inhibitors may lower the virulence of bacterial pathogens. Molecules capable of inhibiting prenylation have been developed for cancer treatment in humans. The potential of these drugs as a new class of broad-spectrum anti-bacterial agents merits further study.

\section{ACKNOWLEDGMENTS}

The author receives support from ANR (reference ANR-09-BLAN-0296-01) and CNRS. He thanks Steve Garvis, Thais L. Lacerda, and Suzana P. Salcedo for proofreading and helpful comments.

\section{REFERENCES}

Al-Quadan, T., and Abu Kwaik, Y. (2011). Molecular characterization of exploitation of the polyubiquitination and farnesylation machineries of Dictyostelium discoideum by the AnkB F-box effector of Legionella pneumophila. Front. Microbiol. 2:23. doi: 10.3389/ fmicb.2011.00023

Boucrot, E., Beuzon, C. R., Holden, D. W., Gorvel, J. P., and Meresse, S. (2003). Salmonella typhimurium SifA effector protein requires its membrane-anchoring C-terminal hexapeptide for its biological function. J. Biol. Chem. 278, 14196-14202.

Campodonico, E. M., Chesnel, L., and Roy, C. R. (2005). A yeast genetic system for the identification and characterization of substrate proteins transferred into host cells by the Legionella pneumophila Dot/Icm system. Mol. Microbiol. 56, 918-933.

Clarke, S., Vogel, J. P., Deschenes, R. J., and Stock, J. (1988). Posttranslational modification of the Ha-ras oncogene protein: evidence for a third class of protein carboxyl methyltransferases. Proc. Natl. Acad. Sci. U.S.A. 85, 4643-4647.

Dowen, R. H., Engel, J. L., Shao, F., Ecker, J. R., and Dixon, J.E. (2009).A family of bacterial cysteine protease type III effectors utilizes acylation-dependent and -independent strategies to localize to plasma membranes. J. Biol. Chem. 284, 15867-15879.

Geddes, K., Worley, M., Niemann, G., and Heffron, F. (2005). Identification of new secreted effectors in Salmonella enterica serovar Typhimurium. Infect. Immun. 73, 6260-6271.

Ivanov, S. S., Charron, G., Hang, H. C., and Roy, C. R. (2010). Lipidation by the host prenyltransferase machinery facilitates membrane localization of Legionella pneumophila effector proteins. J. Biol. Chem. 285, 34686-34698.

Kenny, B., Devinney, R., Stein, M., Reinscheid, D. J., Frey, E.A., and Finlay, B. B. (1997). Enteropathogenic E. coli (EPEC) transfers its receptor for intimate adherence into mammalian cells. Cell 91, 511-520.

Michaelson, D., Ali, W., Chiu, V. K., Bergo, M., Silletti, J., Wright, L., Young, S. G., and Philips, M. (2005). Postprenylation CAAX processing is required for proper localization of Ras but not Rho GTPases. Mol. Biol. Cell 16, 1606-1616.

Nimchuk, Z., Marois, E., Kjemtrup, S., Leister, R. T., Katagiri, F., and Dangl, J. L. (2000). Eukaryotic fatty acylation drives plasma membrane targeting and enhances function of several type III effector proteins from Pseudomonas syringae. Cell 101, 353-363.

Perez-Sala, D. (2007). Protein isoprenylation in biology and disease: general overview and perspectives from studies with genetically engineered animals. Front. Biosci. 12, 4456-4472.

Price, C. T., Al-Quadan, T., Santic, M., Jones, S. C., and Abu Kwaik, Y. (2010a). Exploitation of conserved eukaryotic host cell farnesylation machinery by an F-box effector of Legionella pneumophila. J. Exp. Med. 207, 1713-1726.

Price, C. T., Jones, S. C., Amundson, K. E., and Abu Kwaik, Y. (2010b). Host-mediated post-translational prenylation of novel Dot/Icm-translocated effectors of Legionella pneumophila. Front. Microbiol. 1:131. doi: 10.3389/fmicb.2010.00131

Reinicke, A. T., Hutchinson, J. L., Magee, A. I., Mastroeni, P., Trowsdale, J., and Kelly, A. P. (2005). A Salmonella 
typhimurium effector protein SifA is modified by host cell prenylation and S-acylation machinery. J. Biol. Chem. 280, 14620-14627.

Salcedo, S. P., and Holden, D. W. (2003). SseG, a virulence protein that targets Salmonella to the Golgi network. EMBO J. 22, 5003-5014.

Schmid, M. C., Scheidegger, F., Dehio, M., BalmelleDevaux, N., Schulein, R., Guye, P., Chennakesava, C. S., Biedermann, B., and Dehio, C. (2006). A translocated bacterial protein protects vascular endothelial cells from apoptosis. PLoS Pathog. 2, e115. doi: 10.1371/ journal.ppat.0020115
Wright, L. P., and Philips, M. R. (2006). Thematic review series: lipid posttranslational modifications. CAAX modification and membrane targeting of Ras. J. Lipid Res. 47, 883-891.

Zhang, F. L., and Casey, P. J. (1996). Protein prenylation: molecular mechanisms and functional consequences. Annu. Rev. Biochem. 65, 241-269.

Zurawski, D. V., Mitsuhata, C., Mumy, K. L., Mccormick, B. A., and Maurelli, A. T. (2006). OspF and OspC1 are Shigella flexneri type III secretion system effectors that are required for postinvasion aspects of virulence. Infect. Immun. 74, 5964-5976.
Received: 28 March 2011; accepted: 30 March 2011; published online: 12 April 2011.

Citation: Méresse S (2011) Is host lipidation of pathogen effector proteins a general virulence mechanism? Front. Microbio. 2:73. doi: 10.3389/fmicb.2011.00073

This article was submitted to Frontiers in Cellular and Infection Microbiology, a specialty of Frontiers in Microbiology. Copyright (C) 2011 Méresse. This is an open-access article subject to a non-exclusive license between the authors and Frontiers Media SA, which permits use, distribution and reproduction in other forums, provided the original authors and source are credited and other Frontiers conditions are complied with. 